\title{
Natural Course of an Untreated Metastatic Perirectal Lymph Node After the Endoscopic Resection of a Rectal Neuroendocrine Tumor
}

\author{
Sang Hyung Kim ${ }^{1}$, Dong-Hoon Yang ${ }^{2}$, Jung Su Lee ${ }^{1}$, Soyoung Park ${ }^{1}$, Ho-Su Lee ${ }^{2}$, Hyojeong Lee ${ }^{2}$, \\ Sang Hyoung Park ${ }^{2}$, Kyung-Jo Kim², Byong Duk Ye², Jeong-Sik Byeon², Seung-Jae Myung ${ }^{2}$, \\ Suk-Kyun Yang ${ }^{2}$, Jin-Ho Kim², Chan Wook Kim³, Jihun Kim ${ }^{4}$ \\ Departments of Internal Medicine ${ }^{I}$, Gastroenterology ${ }^{2}$, Colon and Rectal Surgery ${ }^{3}$, and Pathology ${ }^{4}$, Asan Medical Center, University of Ulsan \\ College of Medicine, Seoul, Korea
}

Lymph node metastasis is rare in small (i.e., $<10 \mathrm{~mm}$ ) rectal neuroendocrine tumors (NETs). In addition to tumor size, pathological features such as the mitotic or Ki-67 proliferation index are associated with lymph node metastasis in rectal NETs. We recently treated a patient who underwent endoscopic treatment of a small, grade 1 rectal NET that recurred in the form of perirectal lymph node metastasis 7 years later. A 7-mm-sized perirectal lymph node was noted at the time of the initial endoscopic treatment. The same lymph node was found to be slightly enlarged on follow-up and finally confirmed as a metastatic NET. Therefore, the perirectal lymph node metastasis might have been present at the time of the initial diagnosis. However, the growth rate of the lymph node was extremely low, and it took 7 years to increase in size from 7 to $10 \mathrm{~mm}$. NETs with low Ki-67 proliferation index and without mitotic activity may grow extremely slowly even if they are metastatic. (Intest Res 2015;13:175-179)

Key Words: Rectum; Neuroendocrine tumor; Lymph node; Metastasis

\section{INTRODUCTION}

Neuroendocrine tumors (NETs) are heterogeneous, and arise from the diffuse neuroendocrine system; they are primarily found in the gastrointestinal and respiratory systems. Rectal NETs arise from L-cells, and they are showing an increasing incidence worldwide. ${ }^{1-3}$ Recent increases in the use of screening colonoscopy may have contributed to the rise in the incidence of rectal NETs and may help detect earlystage tumors. ${ }^{4,5}$ The risk of lymph node metastasis in small

Received July 18, 2014. Revised August 20, 2014.

Accepted September 1, 2014.

Correspondence to Dong-Hoon Yang, Department of Gastroenterology, Asan Medical Center, University of Ulsan College of Medicine, 88 Olympic-ro 43-gil, Songpa-gu, Seoul 138-736, Korea. Tel: +82-2-3010-5809, Fax: +822-3010-6517,E-mail: dhyang@amc.seoul.kr.

Financial support: None. Conflict of interest: None. (i.e., $<10 \mathrm{~mm}$ ) rectal NETs is $2-9.7 \%{ }^{6,7}$ In addition to tumor size, lymphovascular invasion, muscularis propria invasion, and the mitotic and Ki-67 proliferation indices are also associated with lymph node metastasis in rectal NETs, and rectal NETs without lymph node metastasis or its risk factors are potential candidates for endoscopic resection. ${ }^{7-10}$ Therefore, preoperative assessment of any metastatic lesions is necessary to determine the therapeutic options for small rectal NETs. However, it is often difficult to distinguish between benign and metastatic nodes in the case of very small lymph nodes, even in patients with more advanced malignancies such as advanced rectal adenocarcinoma.

Here, we describe an endoscopically treated patient who had a small, grade 1 rectal NET and a tiny perirectal lymph node metastasis that was initially regarded as benign at presentation, but was confirmed as a metastatic lymph node 7 years later.

\footnotetext{
๑ Copyright 2015. Korean Association for the Study of Intestinal Diseases. All rights reserved.

This is an Open Access article distributed under the terms of the Creative Commons Attribution Non-Commercial License (http://creativecommons.org/licenses/by-nc/3.0)

which permits unrestricted non-commercial use, distribution, and reproduction in any medium, provided the original work is properly cited.
} 


\section{CASE REPORT}

A 51-year-old male patient was referred to Asan Medical Center on August 10, 2005 for a rectal NET that was found incidentally on screening colonoscopy. The patient had undergone hemorrhoidectomy in his twenties, but otherwise had no significant past medical history. He was a nonsmoker and non-drinker. His height and weight were 164 $\mathrm{cm}$ and $59.3 \mathrm{~kg}$, respectively. His initial vital signs included a blood pressure of 116/74 mmHg, pulse of 84 beats/minute, respiratory rate of 20 breaths/minute, and body temperature of $36.6^{\circ} \mathrm{C}$.

Physical examination revealed no abnormalities. Laboratory data and the results of urinalysis and stool examination were all within normal limits. Chest and abdominal radi- ography were also unremarkable. Colonoscopy revealed a 5-mm-sized subepithelial tumor in the rectum, $5 \mathrm{~cm}$ above the anal verge; histological analysis of a forceps biopsy specimen revealed a rectal NET that was positive for both synaptophysin and chromogranin. Abdominopelvic CT showed no definite rectal lesions, but did reveal a 7-mm-sized perirectal lymph node. Considering the small size of the lymph node and the rare incidence of small metastatic rectal NETs, the malignant potential of the perirectal lymph node was regarded as very low. We discussed the CT findings with the patient, and finally decided to first remove the primary rectal NET endoscopically, with more invasive surgery to be considered if histological analysis revealed any other unfavorable findings, such as lymphovascular invasion, $\geq 2$ mitoses per 50 high-power fields, and/or Ki-67 index $>2 \%$.
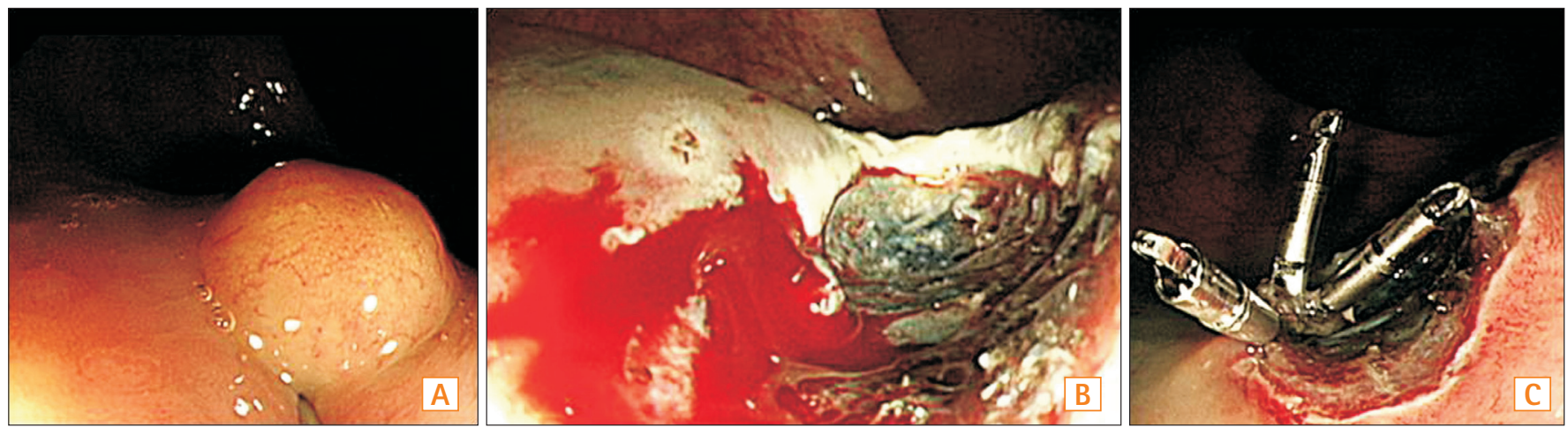

Fig. 1. Endoscopic findings. (A) A 5-mm-sized subepithelial tumor in the rectum. (B and C) Endoscopic mucosal resection was performed.
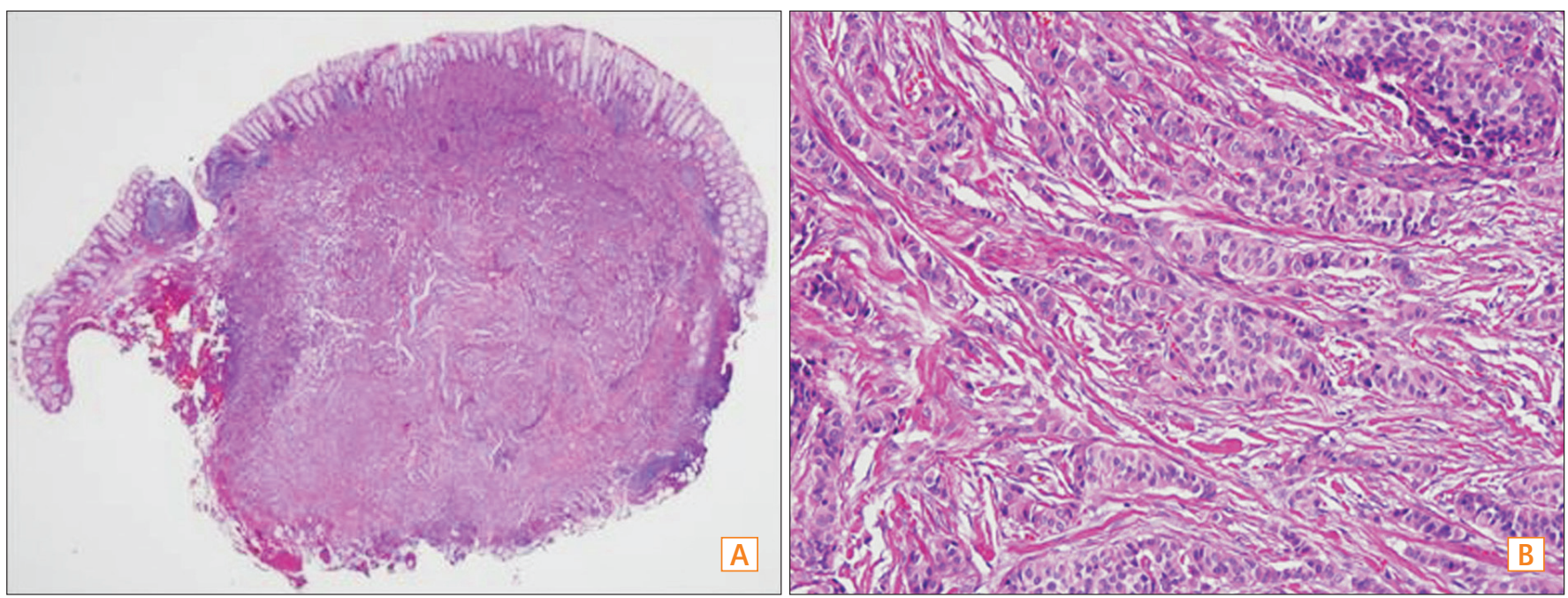

Fig. 2. Histopathological findings of the rectal neuroendocrine tumor. (A) The endoscopically resected specimen mostly consisted of a relatively welldemarcated tumor. The tumor involved the mucosa and submucosa, and it measured $8 \mathrm{~mm}$ across the greatest dimension. The deep resection margin characterized the tumor $(\mathrm{HEE}, \times 10)$. (B) The tumor cells formed nests or cords in the sclerotic stroma and demonstrated histological patterns typical of a neuroendocrine tumor. The tumor cell nuclei are round or ovoid and demonstrate fine salt-and-pepper chromatin (H\&E, $\times 200)$. 
Endoscopic mucosal resection was performed (Fig. 1). The tumor measured $8 \times 5 \times 5 \mathrm{~mm}$, and the vertical resection margin was positive. The tumor involved the mucosa and submucosa without angioinvasion. The Ki-67 index was $0.8 \%$. Hence, the lesion was diagnosed as a well-differentiated grade 1 NET (Fig. 2). Given the favorable histological
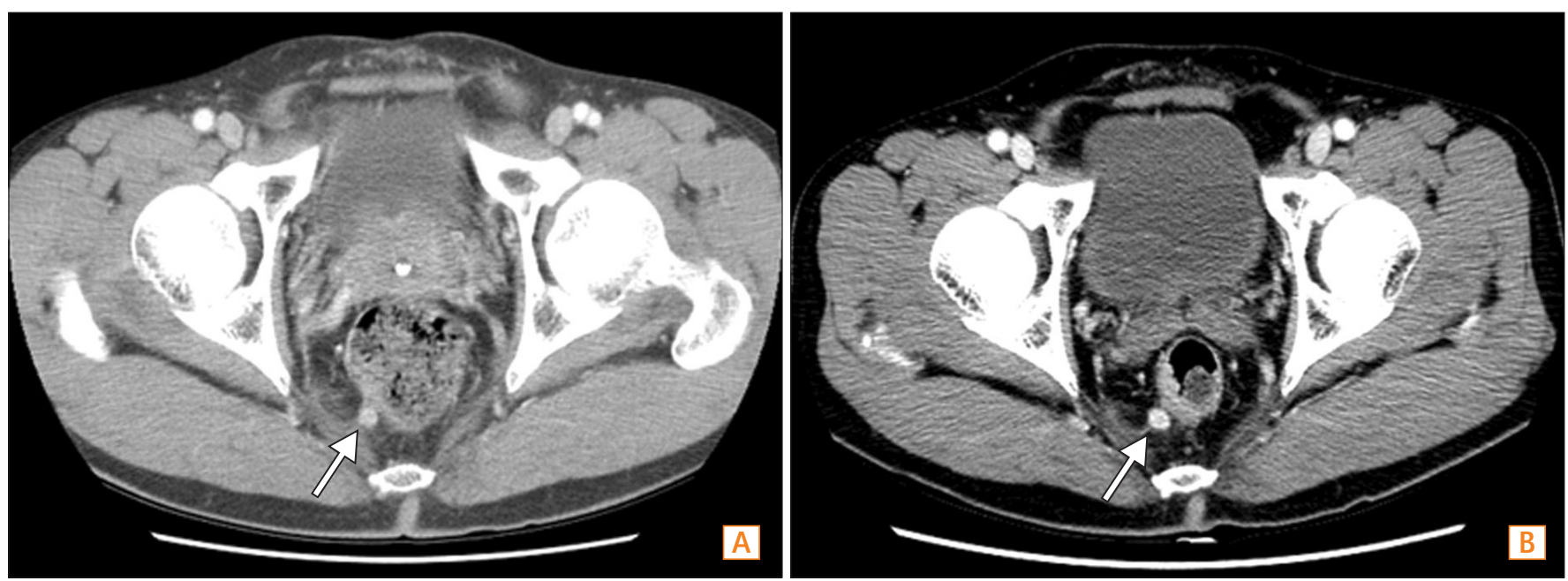

Fig. 3. Abdominopelvic CT findings. (A) The initial CT imaging showed perirectal lymph node. (B) Seven years later, the perirectal lymph node had slightly enlarged from 7 to $10 \mathrm{~mm}$.
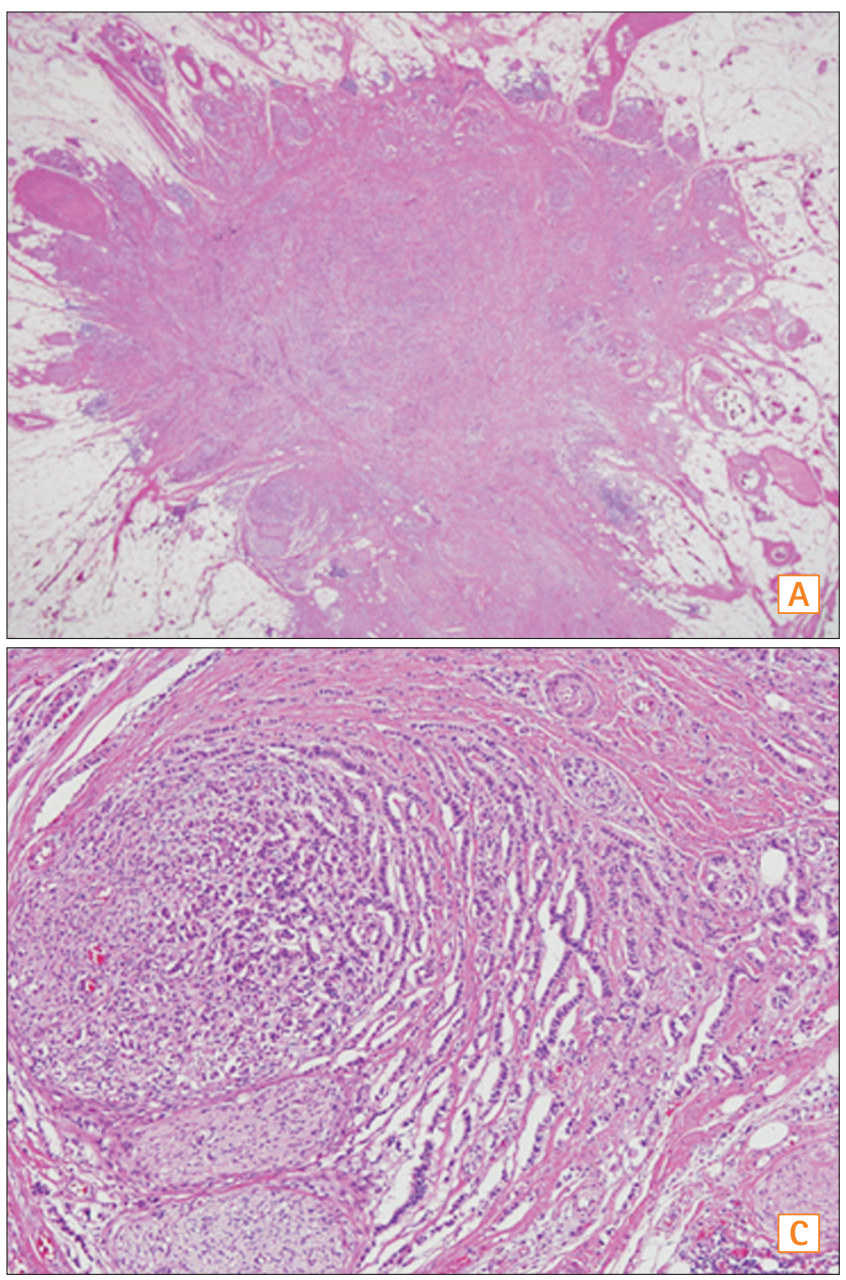

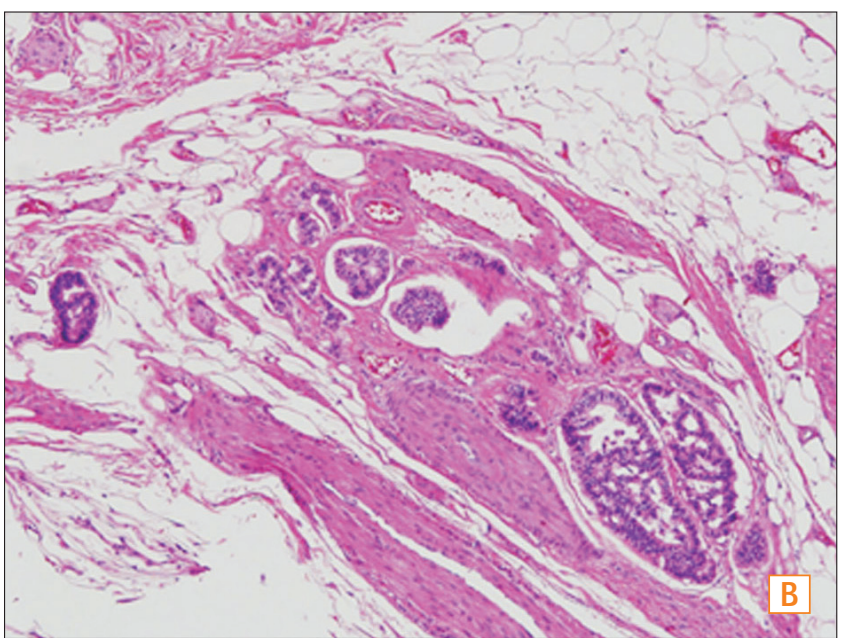

Fig. 4. Histological findings of the perirectal lymph node. (A) The metastatic node was located in the pericolic adipose tissue, but there was no recognizable lymph node structure. The node demonstrated a stellate shape $\left(H \& E_{1} \times 10\right)$. (B) Lymphovascular invasion was noted in the peripheral part of the metastatic node $(\mathrm{HEE}, \times 100)$. (C) Some parts of the tumor demonstrated neural and perineural invasion $\left(H \& E_{1} \times 100\right)$. 
results of the resected specimen, we considered metastasis to the perirectal lymph nodes to be extremely unlikely; thus, we elected to follow-up the patient using endoscopy (annual sigmoidoscopy for the first 3 years, then colonoscopy every 3 years) and annual abdominopelvic CT.

For up to 7 years after endoscopic resection, the size of the perirectal lymph node remained unchanged on CT, and no intraluminal recurrence was identified on serial endoscopic follow-up examinations. However, 7 years after the resection, the lymph node was found to have enlarged slightly, from 7 to $10 \mathrm{~mm}$ (Fig. 3), although colonoscopy revealed no evidence of recurrence in the rectum. Transrectal ultrasoundguided biopsy was performed on the perirectal lymph node, and histological analysis revealed a well-differentiated NET. Preoperative MRI showed two perirectal lymph nodes suspicious for metastasis. Laparoscopic low anterior resection and lymph node dissection were also performed. The surgical specimen contained two metastatic lymph nodes, measuring $10 \times 8 \times 8 \mathrm{~mm}$ and $6 \times 5 \times 5 \mathrm{~mm}$, respectively. Although lymphovascular and perineural invasion were present, the resection margins were clear. The specimen was positive for synaptophysin, but negative for chromogranin. The Ki-67 labeling index was $\leq 2 \%$, but no mitosis was noted. Finally, the surgical specimen was diagnosed as a metastatic, well-differentiated, grade 1 NET (Fig. 4). The patient was discharged without complications and was scheduled to continue to receive follow-up examinations on a regular basis.

\section{DISCUSSION}

Using studies that correlate NET prognosis, the Ki-67 proliferation index, and the mitotic count, the NET classifications of the World Health Organization (WHO) were updated in 2010. ${ }^{11}$ Although the natural course of rectal NETs is not fully understood, recent studies suggest that the WHO classification system correlates well with the metastatic potential and prognosis of rectal NETs. ${ }^{9,12}$ Moreover, recent Korean studies suggest that the endoscopic treatment of small (i.e., $<10 \mathrm{~mm}$ ) rectal NETs without evidence of regional or distant metastasis can achieve highly favorable long-term outcomes. ${ }^{13,14}$ Another recent Korean study suggested that the risk of recurrence is markedly increased in rectal NET patients with metastatic lymph nodes, even after radical surgery (hazard ratio, 12.8; 90\% CI, 4-41 on univariate analysis), although this study did not investigate the histological grade of NETs. ${ }^{15}$ Therefore, before deciding on therapy for a small rectal NET, the presence of metastatic lesions should be investigated by using various imaging modalities, such as
CT, MRI, or endoscopic ultrasonography. However, all these imaging modalities have demonstrated limited diagnostic accuracy for assessing perirectal metastatic lymph nodes. ${ }^{16}$ Although indium-111 pentetreotide scintigraphy remains the gold standard for the diagnosis and localization of most NETs, its utility in colorectal NETs has not been validated owing to the sparse data available, and it may be more difficult to detect lesions of these types because of the greater background activity in the colon and rectum. ${ }^{17}$ Fludeoxyglucose (FDG) used in PET accumulates only in high-grade NETs, ${ }^{18}$ and therefore, FDG-PET has not been considered for imaging in low-grade NETs. Endoscopic ultrasound-guided fine needle aspiration (EUS-FNA) for perirectal lesions could be another option to confirm perirectal lymph node metastasis, but it was not a well-established diagnostic procedure for perirectal lesions less than $10 \mathrm{~mm}$ in size in 2005, when the patient described in the current report was treated. ${ }^{19} \mathrm{In}$ our case, a small NET was noted in the distal rectum and a 7-mm-sized perirectal lymph node was also identified. As mentioned above, it was highly difficult to assess the histological nature of the lymph node without performing surgery at that time. Meanwhile, radical surgery for distal rectal lesions carries the potential risks of bladder or bowel dysfunction and stoma formation. ${ }^{20}$ Therefore, after discussion with the patient, we decided to first perform endoscopic resection of the rectal NET. After removal of that lesion, we discussed the favorable histology of the resected specimen, as well as the question of the unresected small perirectal lymph node with uncertain histology, and the patient finally elected a course of follow-up without invasive surgery.

Because the size of the initially noted lymph node remained unchanged for up to 7 years of follow-up examinations, our initial belief was that this lymph node was benign. However, 7 years and 1 month after the initial local excision, the lymph node was found to have slightly enlarged, and it was finally diagnosed as a metastatic, grade 1 NET. If the initial perirectal lymph node was a metastasis from the rectal NET, this case suggests that metastatic lesions from grade 1 rectal NET might demonstrate an extremely slow growth rate. Therefore, any patients with small, grade- 1 , rectal NETs that are locally excised should receive long-term follow-up examinations when lesions are suspected to be metastatic but are too small for the performance of EUS-FNA for histological confirmation. Highly aggressive approaches such as surgical lymph node dissection can also be considered. However, considering the operation-related complications, the extremely slow growth rate, and the very low incidence of lymph node metastasis from small rectal NETs, surgical 
excision might be substituted by EUS-FNA or by regular follow-up with imaging modalities if EUS-FNA is impossible or does not help in the diagnosis. However, little is known about the adequate follow-up interval in these kinds of situations. On the other hand, if the locally excised primary lesions demonstrate grade 2 histology and any lesions suspected of metastasis are present, either EUS-FNA or surgical excision should be considered to rule out the possibility of metastasis. FDG-PET may also be useful for the staging of grade 2 or 3 NETs compared with grade 1 NETs. ${ }^{18}$

Generalization of the information presented in this extremely rare case should be avoided. Nonetheless, to the best of our knowledge, the natural course of untreated perirectal lymph node metastasis from a grade 1 rectal NET has never been previously described. Thus, this case discussed here can help clinicians understand the nature of metastatic lesions from small, grade 1 rectal NETs.

\section{REFERENCES}

1. Lawrence B, Gustafsson BI, Chan A, Svejda B, Kidd M, Modlin IM. The epidemiology of gastroenteropancreatic neuroendocrine tumors. Endocrinol Metab Clin North Am 2011;40:1-18.

2. Ellis L, Shale MJ, Coleman MP. Carcinoid tumors of the gastrointestinal tract: trends in incidence in England since 1971. Am J Gastroenterol 2010;105:2563-2569.

3. Tsai HJ, Wu CC, Tsai CR, Lin SF, Chen LT, Chang JS. The epidemiology of neuroendocrine tumors in Taiwan: a nation-wide cancer registry-based study. PLoS One doi:10.1371/journal. pone.0062487. Published online 22 April 2013.

4. Ito T, Sasano H, Tanaka M, et al. Epidemiological study of gastroenteropancreatic neuroendocrine tumors in Japan. J Gastroenterol 2010;45:234-243.

5. Scherubl H. Rectal carcinoids are on the rise: early detection by screening endoscopy. Endoscopy 2009;41:162-165.

6. Mani S, Modlin IM, Ballantyne G, Ahlman H, West B. Carcinoids of the rectum. J Am Coll Surg 1994;179:231-248.

7. Soga J. Early-stage carcinoids of the gastrointestinal tract: an analysis of 1914 reported cases. Cancer 2005;103:1587-1595

8. Weinstock B, Ward SC, Harpaz N, Warner RR, Itzkowitz S, Kim MK. Clinical and prognostic features of rectal neuroendocrine tumors. Neuroendocrinology 2013;98:180-187.
9. Jernman J, Valimaki MJ, Louhimo J, Haglund C, Arola J. The novel WHO 2010 classification for gastrointestinal neuroendocrine tumours correlates well with the metastatic potential of rectal neuroendocrine tumours. Neuroendocrinology 2012;95:317324.

10. Hotta K, Shimoda T, Nakanishi Y, Saito D. Usefulness of Ki67 for predicting the metastatic potential of rectal carcinoids. Pathol Int 2006;56:591-596.

11. Bosman FT, Carneiro F, Hruban RH, Theise ND. WHO classification of tumours of the digestive system. 4th ed. Lyon: IARC Press, 2010.

12. Lee JL, Yu CS, Kim M, Hong SM, Lim SB, Kim JC. Prognostic impact of diagnosing colorectal neuroendocrine carcinoma using the World Health Organization 2010 classification. Surgery 2014;155:650-658.

13. Kim GU, Kim KJ, Hong SM, et al. Clinical outcomes of rectal neuroendocrine tumors $\leq 10 \mathrm{~mm}$ following endoscopic resection. Endoscopy 2013;45:1018-1023.

14. Kim DH, Lee JH, Cha YJ, et al. Surveillance strategy for rectal neuroendocrine tumors according to recurrence risk stratification. Dig Dis Sci 2014;59:850-856.

15. Kim MS, Hur H, Min BS, Baik SH, Lee KY, Kim NK. Clinical outcomes for rectal carcinoid tumors according to a new (AJCC 7th edition) TNM staging system: a single institutional analysis of 122 patients. J Surg Oncol 2013;107:835-841.

16. Bipat S, Glas AS, Slors FJ, Zwinderman AH, Bossuyt PM, Stoker J. Rectal cancer: local staging and assessment of lymph node involvement with endoluminal US, CT, and MR imaging-a metaanalysis. Radiology 2004;232:773-783.

17. Mandair D, Caplin ME. Colonic and rectal NET's. Best Pract Res Clin Gastroenterol 2012;26:775-789.

18. Adams S, Baum R, Rink T, Schumm-Drager PM, Usadel KH, Hor G. Limited value of fluorine-18 fluorodeoxyglucose positron emission tomography for the imaging of neuroendocrine tumours. Eur J Nucl Med 1998;25:79-83.

19. Sasaki Y, Niwa Y, Hirooka Y, et al. The use of endoscopic ultrasound-guided fine-needle aspiration for investigation of submucosal and extrinsic masses of the colon and rectum. Endoscopy 2005;37:154-160.

20. Bakker IS, Snijders HS, Wouters MW, et al. High complication rate after low anterior resection for mid and high rectal cancer; results of a population-based study. Eur J Surg Oncol 2014;40:692-698. 